\title{
Nuestros primeros aportes en Salud y Educación
}

\author{
Our first contributions in Health and Education
}

Valles-Coral, Miguel Angel ${ }^{1[0000-0002-8806-2892]}$

${ }^{1}$ Universidad Nacional de San Martín, Tarapoto, Perú mavalleseunsm.edu.pe

\section{Editorial}

La Universidad Nacional de San Martín, desde la obtención del licenciamiento y a pesar de las duras condiciones restrictivas impuestas por el estado a consecuencia de la pandemia generada por el COVID 19 (BBC, 2020), viene trabajando arduamente para consolidar las condiciones básicas de calidad que permitan formar profesionales altamente calificados, que respondan a las exigencias de las comunidades de su área de influencia, teniendo la misión de generar desarrollo socio económico que permita mejorar las condiciones de vida de la población.

En ese sentido, las autoridades universitarias hicieron una fuerte apuesta para consolidar la posición que viene obteniendo como ente académico, científico y de investigación de referencia de la macro región oriente. Prueba de ello es que en el II Informe Bienal Sobre la Realidad Universitaria en el Perú (SUNEDU, 2020), la UNSM aparece en los rankings de producción científica gracias a la publicación de artículos científicos en revistas indexadas por parte de los docentes (Cáceres, 2014), dejando de ser una universidad más, posicionándose mejor en estos y otros rankings. Así lo demuestra el estudio de (Valles-Coral et al., 2020) donde la universidad pasó del puesto 64 en el año 2018 hasta el 44 en el 2020 (Aguillo, 2020), mejorando 20 posiciones; siendo la universidad nacional que mejor desempeño ha mostrado en ese periodo.

Es así que el Vice Rectorado de Investigación en coordinación estrecha con el Fondo Editorial, la Decanatura y la Unidad de Investigación de la Facultad de Ingeniería de Sistemas e Informática, durante el segundo semestre de 2020 ha venido trabajando en la creación y gestación de la primera Revista Científica de Sistemas e Informática que cumple con las exigencias para lograr su indexación en índices importantes como DOAJ, Redalyc, Latindex Directorio y Scielo.

Ponemos entonces a disposición de la comunidad universitaria el volumen 1, número 1, donde realizamos nuestros primeros aportes en salud y educación a fin de que los artículos sean revisados y tengan el impacto que esperamos en la comunidad científica, haciendo votos para que este proyecto se consolide en el mediano plazo y con ello se consolide el posicionamiento de la Universidad Nacional de San Martín.

Universitariamente.

\section{Ing. Dr. Miguel Ángel Valles Coral Investigador Calificado RENACYT - CONCYTEC Director del Fondo Editorial Universidad Nacional de San Martín}




\section{Referencias bibliográficas}

Aguillo, I. (2020). Peru | Ranking Web of Universities: More than 28000 institutions ranked. Retrieved February 27, 2019, from http://www.webometrics.info/en/Latin_America/Peru

BBC. (2020). Coronavirus: Perú decreta cuarentena general en el país y el cierre de fronteras durante 15 días ante la pandemia de covid-19 - BBC News Mundo. Retrieved April 18, 2020, from https://www.bbc.com/mundo/noticias-america-latina-51902989

Cáceres, G. (2014). La importancia de publicar los resultados de Investigación. Revista Facultad de Ingeniería, 23(37), 7-8. $\quad$ Retrieved from http://www.scielo.org.co/scielo.php?script=sci_arttext\&pid=S0121-11292014000200001

SUNEDU. (2020). II Informe Bienal sobre la Realidad Universitaria en el Perú | Gobierno del Perú. Retrieved January 5, 2021, from https://www.gob.pe/institucion/sunedu/informespublicaciones/1093280-ii-informe-bienal-sobre-la-realidad-universitaria-en-el-peru

Valles-Coral, M. A., Riascos-Armas, J. O., and Hernandez-Torres, E. A. (2020). Management of the digital identity of researchers and its effect on the webometrics ranking of a peruvian amazon university. Revista Cubana de Informacion En Ciencias de La Salud, 31(2). https://doi.org/10.36512/rcics.v31i1.1406 